\title{
MOBILE SURVEILLANCE SENSOR NETWORK
}

\author{
Peter Bernhardt, Michael Hendrix
}

\section{Zusammenfassung}

Der Schutz von Personen, Veranstaltungen, Einrichtungen und Einzelobjekten muss den gesellschaftlichen und globalen Wirklichkeiten stetig angepasst werden, um seine Wirksamkeit nicht zu verlieren. Dieser Artikel präsentiert ein mobiles Überwachungsnetzwerk, welches eine Entwicklung zur Absicherung von Schutzobjekten, insbesondere von Gemälden, und zur Bereitstellung einer Lageübersicht für Sicherheitskräfte an nicht veränderbaren oder temporären Standorten darstellt. Die Sensoren des Systems sind manipulationsresistent, das heißt sie benötigen keine Ressourcen von der Umgebung und erfordern keine permanenten Veränderungen am Einsatzort. Im Rahmen von Feldversuchen während der Entwicklung wurde das System entsprechend realer Anforderungen kontinuierlich evaluiert und letztendlich die Wirksamkeit der Ergebnisse aufgezeigt.

\section{Abstract}

The protection of persons, events, institutions and individual objects must be adapted to the social and global realities constantly in order not to lose its effectiveness. This article presents a mobile surveillance network, which is a development for the protection of safety objects, especially paintings, and to provide a situational awareness of the security forces of non-modifiable or temporary sites. The sensors of the system are tamper-resistant, which means they do not require resources from the environment and do not require permanent changes in the field. In the context of field trials during the development the system was continuously evaluated according to real requirements and ultimately demonstrated the effectiveness of the results.

\section{EINFÜHRUNG}

Der Schutz von Personen, Veranstaltungen, Einrichtungen und Einzelobjekten bekommt durch die gesellschaftliche und globale Wirklichkeit eine immer höhere Priorität. Dieses weltweit gestiegene Bedürfnis nach Sicherheit sorgt für einen Schub in der Entwicklung innovativer Sicherheitstechnologien. Nach einer in Auftrag gegebenen Studie des BMWI aus dem Jahre 2013 [1] wächst der weltweite Markt für zivile Sicherheitstechnik zwischen 2006 und 2015 von 55 Mrd. Dollar auf 231 Mrd. Dollar pro Jahr. Einem Gutachten der VDI/VDE Innovation und Technik GmbH zufolge weist der Markt für Sicherheitstechnologien und-dienstleistungen in Deutschland auf längere Sicht überdurchschnittliche Wachstumsperspektiven auf. Allein in Deutschland ist von einer Steigerung des Gesamtumsatzes von 20 Mrd. auf 31 Mrd. Euro zwischen 2008 und 2015 auszugehen [2].

Einer Pressemitteilung der Gütegemeinschaft Metallzauntechnik e.V. [3] war zu entnehmen, dass die nichtmilitärische Sicherheitsvorsorge - in erster Linie physische Sicherheitssysteme und -produkte, Identifikation und Authentifizierung, Kontrollen und Gefahrerkennung, Schutz von kritischen Infrastrukturen oder von Lieferketten, Systeme und Produkte zur Kommunikation über Gefährdungen - ein gewaltiger Markt sei und darüber hinaus ein ausgesprochenes Wachstumssegment. Quantitative Aussagen zum Marktsegment „Perimeterschutz“ liegen aber nicht vor.

Die Anwendung von stationären, elektronischen Sicherheitseinrichtungen im Rahmen dieser Zielstellung ist teuer und kompliziert. Eine temporäre Installation von solcherlei Maßnahmen zum zeitweisen Schutz oben genannter Subjekte verbietet sich in der Regel aufgrund des Missverhältnisses von Investitionshöhe zu Nutzungsdauer. Dies liegt im Wesentlichen auch daran, dass die heute angebotenen Systeme ressourcenhungrig sind und/oder Veränderung an der Umgebung notwendig machen. Einen guten Überblick über heutzutage angebotene Systeme zum Primeterschutz erhält man im Online-Portal zu Sicherheitstechnik [4]. Spezielle Informationen zum Pe- rimeterschutz durch mobile Wärmebildkameras finden sich in [5].

Im Rahmen des Vorhabens „Mobile Surveillance Sensor Network" wurde ein System zur temporären Absicherung von Schutzobjekten und zur Bereitstellung einer Lageübersicht (situational awareness) für Sicherheitskräfte an nicht vordefinierten Standorten entwickelt. Dies bedeutet, dass die Teile des Systems keine Ressourcen von der Umgebung benötigen und dadurch unabhängig von ihr funktionieren sowie dass die Installation in einem zeitlich übersichtlichen Rahmen durchgeführt werden kann und keine permanenten Veränderungen an der Umgebung verlangt.

Des Weiteren ist das entwickelte Sensornetzwerk im Unterschied zu den auf dem Markt vorhandenen Angeboten zur Umfeldsicherung konsequent nicht-stationär ausgelegt und somit überall schnell als auch mit vergleichsweise geringem Aufwand einsetzbar. 


\section{GRUNDAUFBAU DES SYSTEMS}

Das System besteht aus verschiedenartigen mobilen Sensoren, welche mit Hilfe der Nutzung einer proprietären Funktechnologie robust und sicher (manipulations- als auch abhörsicher) zu einem Sicherheitsnetz miteinander verbunden werden können. Der Kern des Systems ist eine zentrale Basisstation (Leitstand), die aus einer intelligenten Verknüpfung der verschiedenen Sensorsignale spezifische Bedrohungsmuster extrahiert, wodurch eventuelle Gefahren frühzeitig und sicher erkannt werden können. Die Verknüpfung unterschiedlicher Sensorsignale führt in vielen Szenarien erst zu einer ausreichend hohen Erkennungswahrscheinlichkeit bei gleichzeitiger verringerter Falschalarmrate. Zusätzlich zur Analyse von Bedrohungsmustern ist die Basisstation auch für die Überwachung der Systemintegrität zuständig. Im Falle einer potenziellen Bedrohung werden Sicherheitskräfte mittels Mobilfunk auf ihren mobilen Endgeräten informiert.

Die konzeptionelle Basis des Systems sind zwei durch eine Basisstation miteinander verbundene Kommunikationsnetzwerke. Abbildung 1 zeigt den Grundaufbau des Systems.

Die Kommunikation zwischen Sensormodul und Basisstation erfolgt über eine proprietäre Funktechnologie auf dem $868 \mathrm{MHz}$ Band. Die Kommunikation zwischen Basisstation und einem mobilem Endgerät erfolgt mittels TCP/ IP über GSM/GPRS/UMTS. Jegliche Kommunikation zwischen Sender und Empfänger ist gegen Manipulation und Abhörbarkeit abgesichert.

\section{SENSORMODULE}

Auf der Seite der Datenerhebung befindet sich eine Zahl von Sensormodulen mit einer jeweils hohen Spezialisierung hinsichtlich der zu überwachenden Parameter. Die Sensormodule sind voneinander komplett unabhängig und ebenso nicht mit der zu überwachenden Umgebung abhängig verbunden. Dies bedeutet, dass die Funktionsfähigkeit der Sensoren an sich nicht von durch die Umgebung bereitgestellten Ressourcen abhängig ist (insbesondere Stromversorgung durch Einsatz einer Batterie) und somit eine Position als möglichst "objektiver Beobachter“ effektiv eingenommen werden kann. Die zusätz- habung, Fixierungsmöglichkeiten) gestaltet sind, andererseits die effiziente Anwendung und Konfiguration durch den Nutzer flexibel und möglichst ohne Hilfsmittel erfolgen kann.

Der Messwert eines Sensors wird in regelmäßigen Abständen ausgelesen und einer Toleranzbereichsbetrachtung unterzogen. Beim Toleranzbereich handelt es sich nicht um fest definierte Grenzwerte, vielmehr sind die Grenzwerte dynamisch und relativ zum aktuellen Messwert. So besagt bspw. eine konfigurierte Toleranz von $10 \%$, dass bei einem eingeschwungenen Messwert 100 alle aktuellen Messwerte innerhalb von 90 bis 110 zulässig sind. Sofern der aktuelle Messwert außerhalb des Toleranzbereichs liegt, wird eine verschlüsselte Alarmnachricht erstellt und an die Basisstation verschickt. Abbildung 2 verdeutlich diesen Kernprozess innerhalb eines Sensormoduls.

\section{Sensormodul}

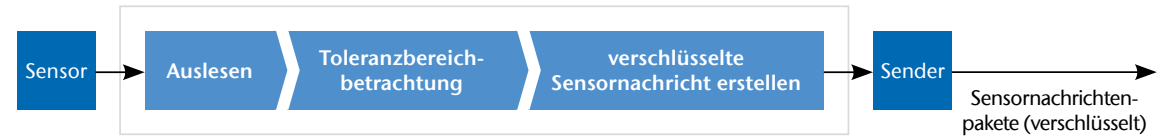

Abb. 2) Schematische Darstellung des Kernprozesses im Sensormodul

liche Forderung nach einer hohen Mobilität bedingt weiterhin, dass die Sensormodule in Material und Form einerseits robust für den Einsatz in verschiedensten Umgebungen (Hand-
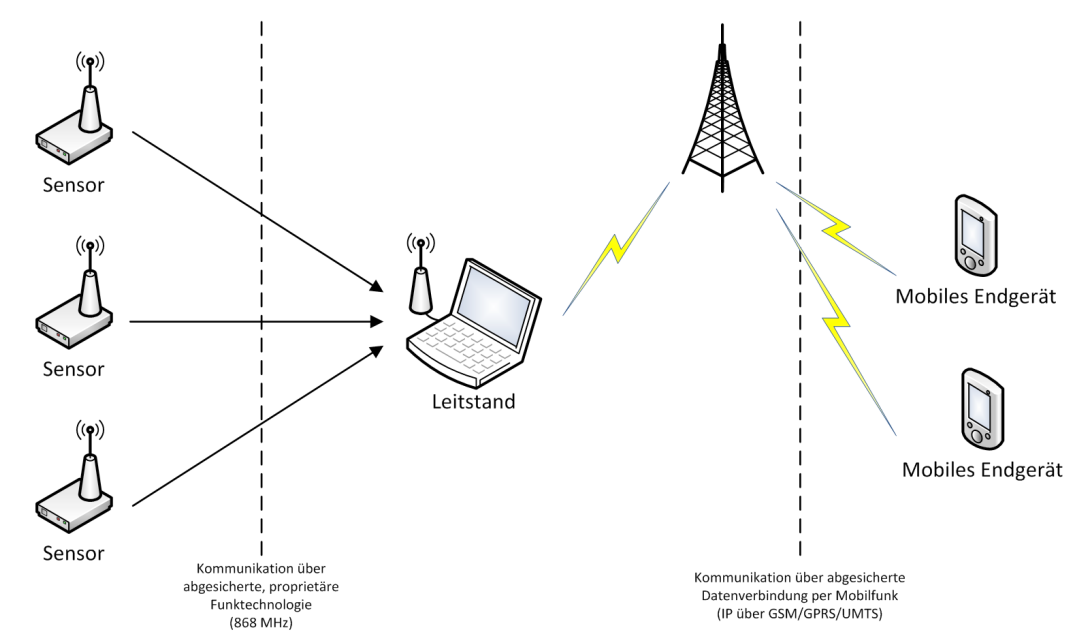

Abb. 1) Grundaufbau des Systems

Neben der Alarmnachricht gibt es noch eine Ping-Nachricht, die in periodischen Abständen an die Basisstation verschickt wird und als Lebenszeichen des Sensormoduls dient. Dadurch kann in der Basisstation bspw. festgestellt werden, ob ein Sensormodul noch über genügend Batteriekapazität verfügt. Zusätzlich wird dadurch das Gesamtsystem mit einem Sabotageschutz versehen, da die Basisstation das dauerhafte Ausbleiben von Nachrichten einzelner oder aller Sensoren erkennen kann und die dadurch entstehende Absicherungslücke ermittelt.

Jede Nachricht des Sensormoduls wird mit einem individuellen Sensormodulpasswort, das nur das Sensormodul und die Basisstation kennen, symmetrisch verschlüsselt. Das Sensormodulpasswort wird während der Initialisierung/ Konfiguration des Sensormoduls in einer kontrollierten Umgebung in diesem hinterlegt und kann nicht ausgelesen werden. 
Der Empfang jeder Nachricht des Sensormoduls muss im Request-Response-Verfahren (siehe u.a. [1], Seite 184) durch die Basisstation unmittelbar bestätigt werden. Andernfalls wird die Nachricht durch das Sensormodul erneut versandt. Dadurch wird der Erfolg des Transports der Informationen vom Sender zum Empfänger sichergestellt, da sonst im Fall einer möglichen Nachrichtenkollision die kollidierten Nachrichten nicht weiterverarbeitet werden könnten und deren Informationen somit verloren gehen würden.

Die Sensoren wurden alle unter der Prämisse einer hohen Detektionsempfindlichkeit bei gleichzeitig sehr niedriger Falschalarmrate entwickelt. Der Aufbau der verschiedenen Sensormodule folgt dabei einem Basisaufbau mit allen Bestandteilen außerhalb des Sensors, welches dann um den jeweiligen Sensor ergänzt wird. Dies ermöglicht ein hohe Kosteneffektivität, eine kompakte Bauweise sowie eine klare Zuordnung einzelner Module für die verschiedenen Einsatzzwecke. Folgende Sensoren wurden entwickelt und hinsichtlich Robustheit und minimalem Energieverbrauch optimiert:

- lichtempfindlicher Sensor, z.B. zur Absicherung des Zugangs zu Gebäuden / Räumen

- Trittmatte, z.B. zur Absicherung des Zugangs zu Gebäuden / Räumen

- neigungsempfindlicher Sensor, z. B. zur Absicherung von Ausstellungsstücken

— intelligente Gemäldeaufhängung zur Absicherung wertvoller Kunstgegenstände

Abbildung 3 zeigt den Basisaufbau eines Sensors am Beispiel des neigungsempfindlichen Sensors.

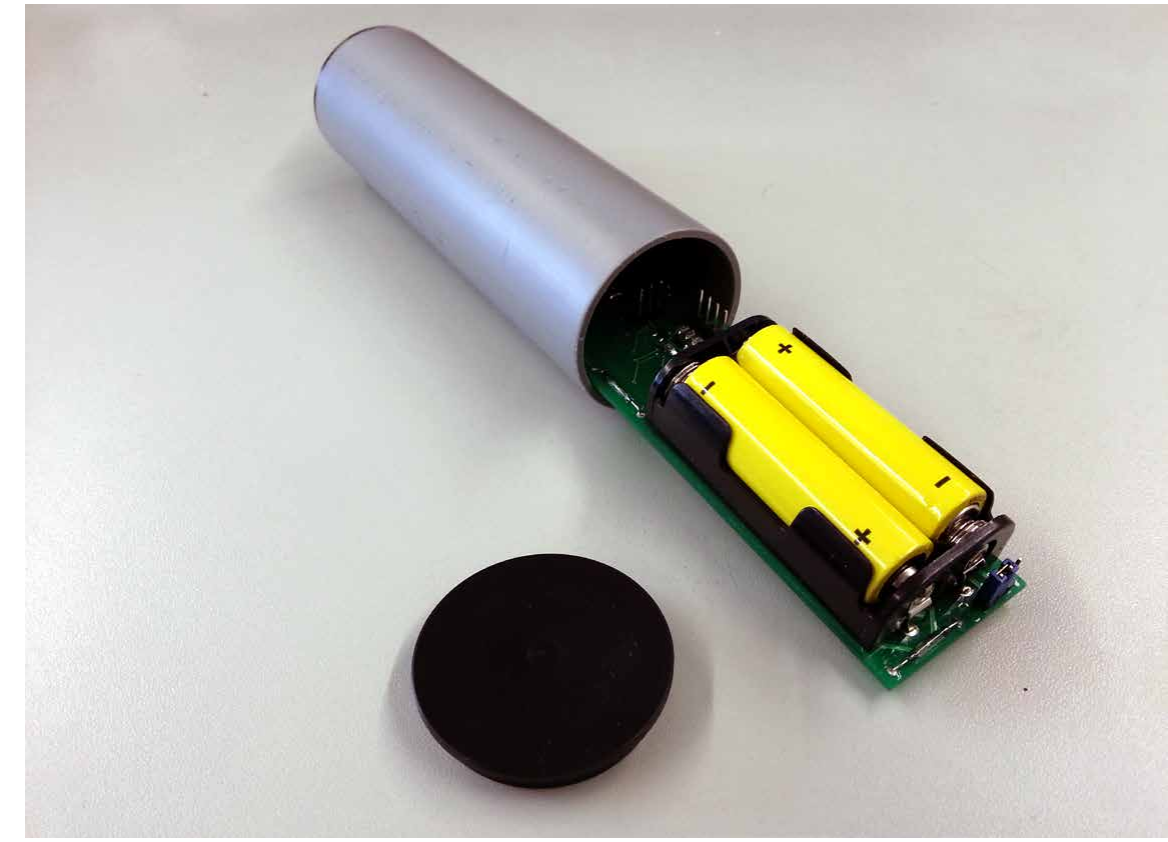

Abb. 3) Neigungsempfindlicher Sensor
Durch den modularen Charakter einer Sensoreinheit hinsichtlich Sensorgehäuse und Kommunikationsprotokoll ist es nicht schwierig, das System bei Bedarf um weitere Sensortypen zu erweitern.

Abbildung 4 zeigt die beispielhaft intelligente Gemäldeaufhängung. Das Stativ ist nicht Teil des Sensorsystems und dient lediglich der Demonstration. Dieser Sensor besitzt eine sehr niedrige Falschalarmrate, reagiert auf einen Gemäldediebstahl dennoch sehr empfindlich.
Die Gemäldeaufhängung kann vom Durchmesser und von der Länge her in bestimmten Grenzen an spezifische Szenarien angepasst werden. Je nach Gewicht des zu schützenden Gemäldes können unterschiedliche Materialien verwendet werden.

Nachfolgende Abbildungen 5 und 6 zeigen die Ergebnisse von Tests zur Messempfindlichkeit der Gemäldeaufhängung. Die Stange bestand aus ferritischem Chromstahl 1.4521 (Viega Sanpress), war $200 \mathrm{~cm}$ lang und hatte einen Durchmesser von $3,5 \mathrm{~cm}$. Ein Messwert wurde als Mittelwert aus drei Einzelmessungen berechnet.

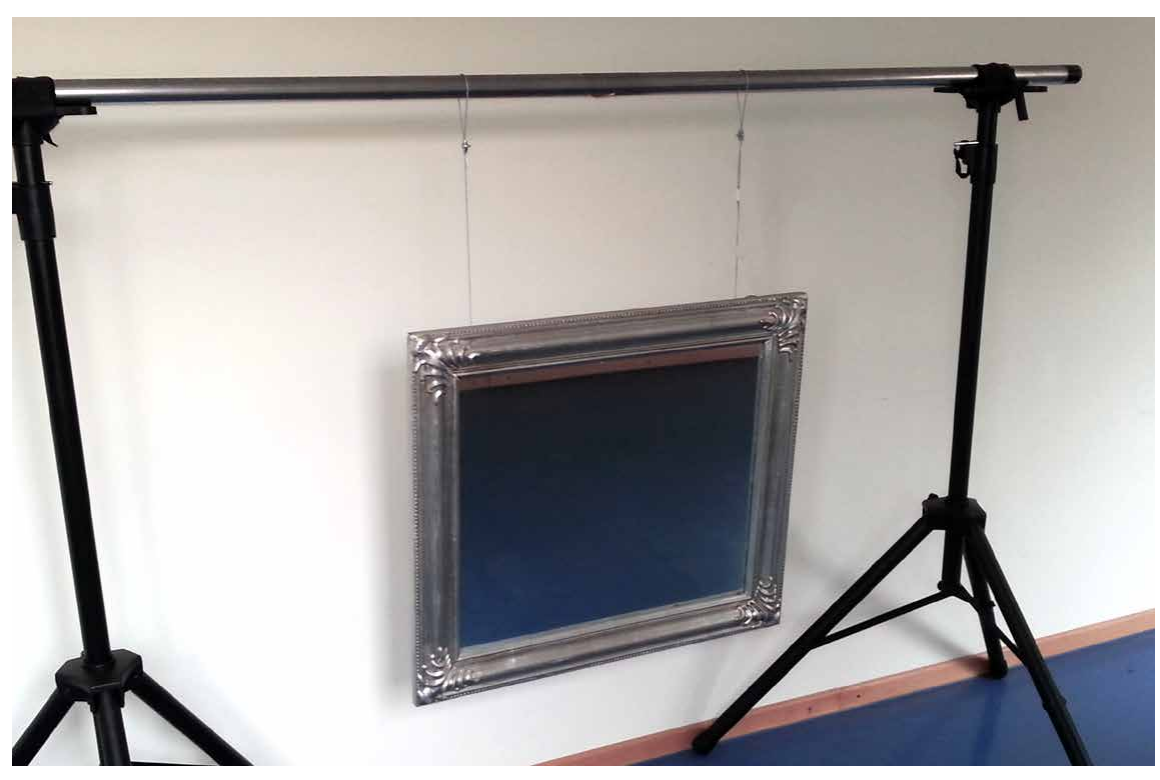

Abb. 4) Intelligente Gemäldeaufhängung 
Die sich deutlich zeigende Linearität der Messwerte weist die Tauglichkeit dieses Verfahrens für die zuverlässige Erkennung von Änderungen der abzusichernden Objekte bzw. deren Masse und vor allem die Adaptierbarkeit auf unterschiedlichste Materialbeschaffenheiten der Schutzobjekte eindeutig nach.

\section{BASISSTATION}

Die Basisstation (Leitstand) ist die zentrale Verwaltungs- und Überwachungseinheit des Systems. Abbildung 7 zeigt eine schematische Darstellung des Kernprozesses in der Basisstation.

Nach dem Empfang einer verschlüsselten Sensornachricht wird diese zunächst entschlüsselt, validiert und in der Datenbank geloggt. Anschließend wird die Sensornachricht an die Mustererkennung weitergeleitet, in der sie mit bereits empfangenen Sensornachrichten gegen hinterlegte Bedrohungsmuster geprüft wird. Wurde ein Muster erkannt, so wird eine Alarmnachricht erstellt und an den Dispatcher weitergeleitet. Im Dispatcher wird anhand einer hinterlegten Eskalationsstrategie entschieden, an wen die Alarmnachricht verschickt werden soll. Abschließend werden alle ausgehenden Alarmnachrichten geloggt und an die mobilen Endgeräte versendet.

Eine eingehende Antwortnachricht von einem mobilen Endgerät wird zunächst geloggt und anschließend an den Dispatcher weitergeleitet. In Abhängigkeit der Antwortnachricht und hinterlegten Eskalationsstrategie wird entschieden, ob eine Alarmnachricht an ein anderes mobiles Endgerät verschickt werden soll.

\section{Messempfindlichkeit für verschiedene Aufhängepunkte}

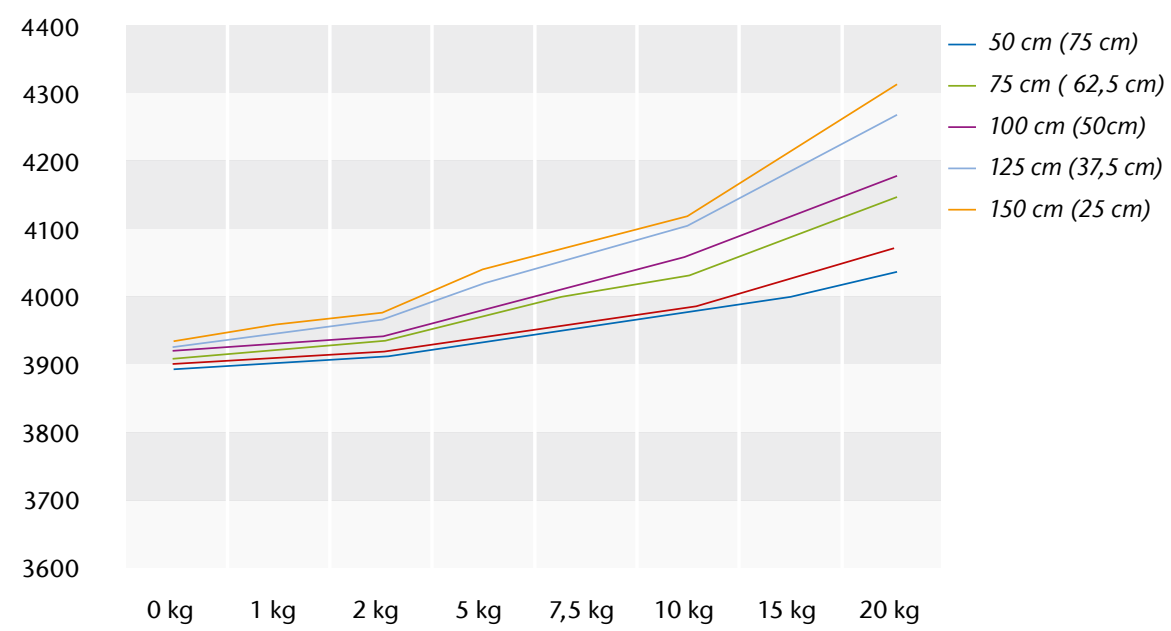

Abb. 5) Messempfindlichkeit für verschiedene Aufhängepunkte.

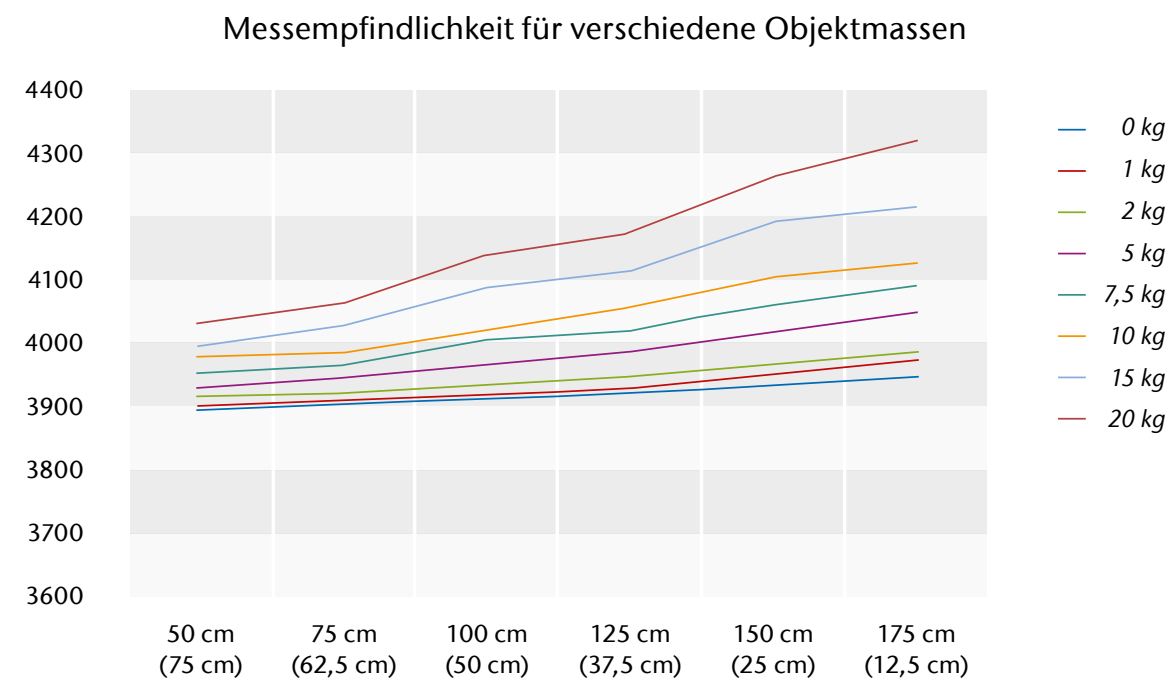

Abb. 6) Messempfindlichkeit für verschiedene Objektmassen.

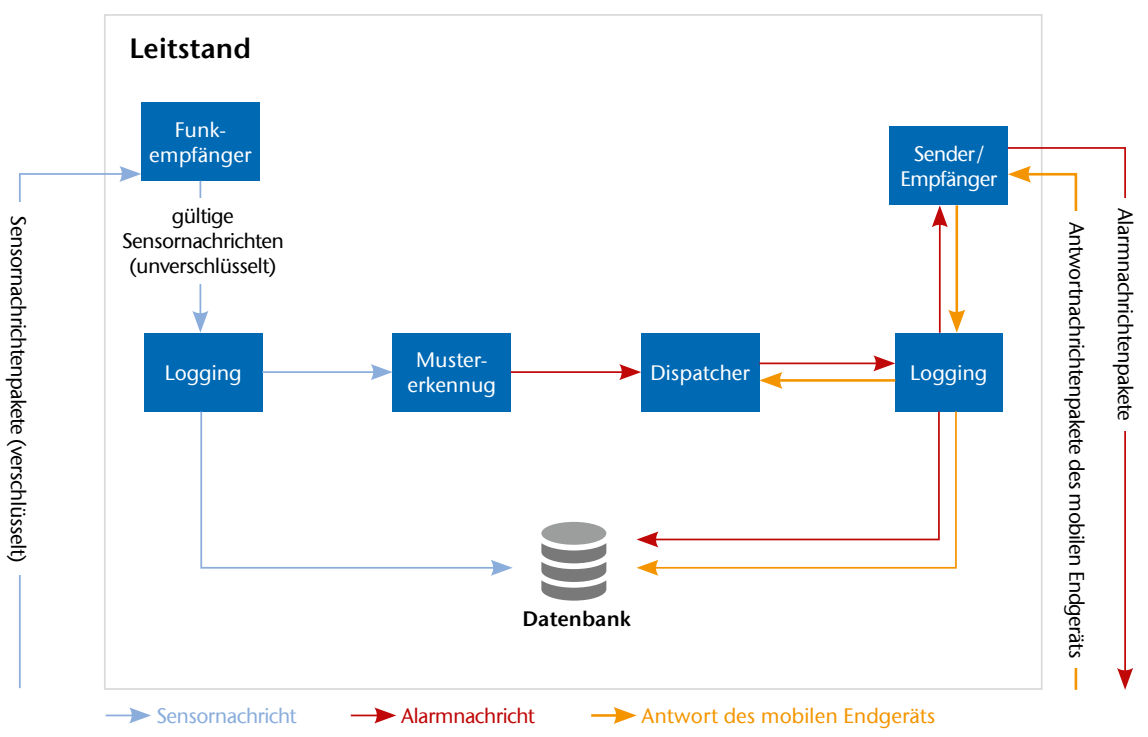

Abb. 7) Schematische Darstellung des Kernprozess in der Basisstation (Leitstand). 
Abbildung 8 zeigt einen Ausschnitt der grafischen Nutzerschnittstelle des Systems.

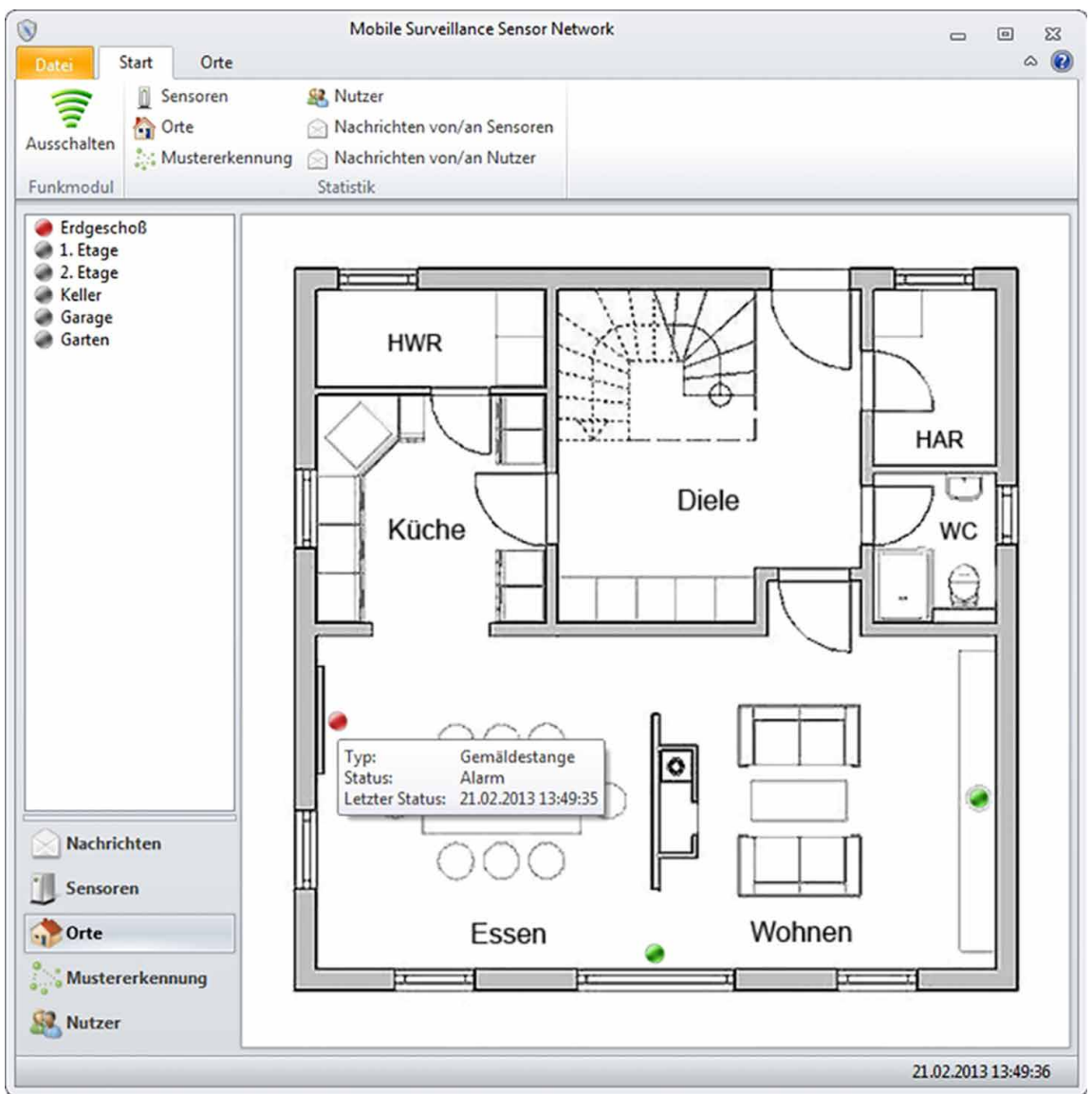

Abb. 8) Grafische Nutzerschnittstelle (Ausschnitt)

Ziel der Entwicklung der grafischen Benutzeroderfläche war es primär, einen Rahmen für die Einrichtung und den Betrieb des Prototyps inklusive der Anbindung von mobilen Endgeräten zu schaffen und damit die Leistungsfähigkeit der Entwicklungsergebnisse unter Bereitstellung einer hohen Benutzbarkeit zu demonstrieren

\section{FAZIT}

Zusammenfassend lässt sich sagen, dass die Kombination aus moderner, besonders stromsparender Informationsverarbeitungstechnik zur Bildung eines robusten Netzwerkes in Verbindung mit dem Konzept eines universellen, modularen Sensorträgers das Ziel erreicht, ein Verfahren zur Absicherung von Personen, Veranstaltungen, Einrichtungen und Einzelobjekten mit sehr guten Leistungsparametern zu schaffen. Dies bestätigte sich auch in Pilotversuchen in realen Anwendungsumgebungen und der Vorstellung der Entwicklung bei den technischen

\section{LITERATUR}

[1] G. Hohpe und B. Woolf, Enterprise Integration Patterns: Designing, Building, and Deploying Messaging Solutions, Boston: Addison-Wesley Professional, 2003.

[2] B. S. Arge IABG, „Forschungsprojekt 26/12 Masterplan Zivile Sicherheitswirtschaft,“ Bundesministerium für Wirtschaft und Technologie, Berlin, 2013.

[3] VSW Verband für Sicherheit in der Wirtschaft Niedersachen e.V., "Marktpotential für Sicherheitstechnologien," 2009.

[4] Gütegemeinschaft Metallzauntechnik, „Pressemitteilung: Markt für Perimeterschutz als Teil der „Global Security“ ist ein Wachstumssegment!,“ 2010.

[5] „Sicherheit.info,“ [Online]. Available: http://www. sicherheit.info. [Zugriff am 201311 25].

[6] Sicherheit durch Technik, "Markt für Wärmebildkameras entwickelt sich,“ [Online]. Available: http:// www.sicherheits-berater.de/schwerpunkthefte/ artikel-ohne-abo/perimeterschutz-ausgabe-12-2010/ markt-fuer-waermebildkameras-entwickelt-sich. html. [Zugriff am 197311 25].

\section{AUTOREN}

Peter Bernhardt, MSIE

Technologietransfer- und Weiterbildungszentrum an

der Technischen Hochschule Wildau [FH] e.V.

Bahnhofstraße, 15745 Wildau

peter.bernhardt@th-wildau.de

Prof. Dr. Michael Hendrix

Technische Hochschule Wildau [FH]

Wirtschaftsinformatik

michael.hendrix@th-wildau.de
Verantwortlichen aus dem angedachten Anwenderkreis. Insbesondere die Unabhängigkeit des Systems von der Umwelt und die lange Laufzeit ohne externen Wartungsbedarf sind die entscheidenden Faktoren zur Abgrenzung gegenüber bestehenden Angeboten. Zusätzlich dazu betont die innovative und ebenfalls minimalinvasive Absicherung von Gemälden die praktische Verwertbarkeit der im Rahmen dieses Forschungsprojektes erzielten Ergebnisse.

\section{DANKSAGUNG}

Der Artikel ist das Ergebnis von Forschungsarbeiten, die im Rahmen eines vom BMWI geförderten Projektes durchgeführt wurden. 